\title{
Universales indiscernibles
}

\author{
Indiscernible Universals
}

\author{
Gonzalo Rodriguez-Pereyra \\ Oriel College, Oxford \\ gonzalo.rodriguez-pereyra@oriel.ox.ac.uk
}

\begin{abstract}
Resumen
Tradicionalmente, se ha pensado que los universales obedecen a la identidad de los indiscernibles, es decir, tradicionalmente se ha pensado que no puede haber universales perfectamente similares. Pero al menos en la concepción de los universales como inmanentes, no hay nada que impida el que haya universales indiscernibles. En este artículo, pretendo argumentar que los universales indiscernibles pueden hacer un trabajo útil, de modo que podría haber razones para postular universales indiscernibles. En particular, argumentaré que postular universales indiscernibles permitiría identificar a una teoría de universales los particulares con bundles de universales, y permitiría también desarrollar una explicación de la semejanza de los universales cuantitativos que evite las objeciones con las que la explicación de Armstrong se enfrenta. Finalmente, responderé algunas de las objeciones y objetaré al criterio de distinción entre particulares y universales de acuerdo con el cual la distinción entre particulares y universales yace en que mientras puede haber particulares indiscernibles, no puede haber universales indiscernibles.
\end{abstract}

Palabras Clave: universales, bundle theory, determinables, semejanza, similitud.

\begin{abstract}
Universals have traditionally thought to obey the identity of indiscernibles, that is, it has traditionally been thought that there can be no perfectly similar universals. But at least in the conception of universals as immanent, there is nothing that rules out there being indiscernible universals. In this paper, I shall argue that there is use-
\end{abstract}

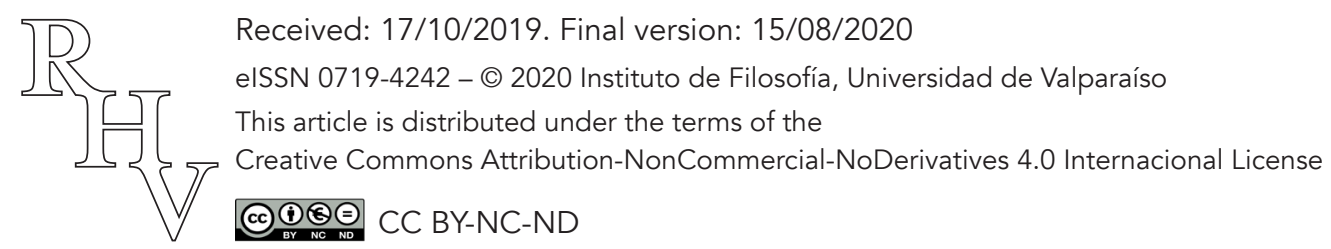


ful work indiscernible universals can do, and so there might be reason to postulate indiscernible universals. In particular, I shall argue that postulating indiscernible universals can allow a theory of universals to identify particulars with bundles of universals, and that postulating indiscernible universals can allow a theory of universals to develop an account of the resemblance of quantitative universals that avoids the objections that Armstrong's account faces. Finally, I shall respond to some objections and I shall undermine the criterion of distinction between particulars and universals that says that the distinction between particulars and universals lies in that while there can be indiscernible particulars, there cannot be indiscernible universals.

Keywords: universals, Bundle Theory, determinables, resemblance, similarity.

\section{Introducción}

La concepción de los universales que tendré en consideración es una concepción de acuerdo con la cual los universales tienen instancias, existen en sus instancias (son inmanentes, universales in rebus) y son completamente idénticos a través de sus instancias. Armstrong y otros han sostenido una concepción tal de los universales (cf. Armstrong 1997, 27, 66).

Por universales indiscernibles entenderé universales perfectamente similares, o perfectamente semejantes (entenderé exactamente lo mismo por "similitud" y "semejanza" y sus respectivas palabras derivadas. Pero, ¿cuándo dos universales son perfectamente similares? Cuando ellos confieren similitud perfecta en un respecto a los particulares que los instancian. Si U y U* son dos universales, $a$ y $b$ son perfectamente similares en su rojez, e instanciar $U$ es lo que hace que $a$ sea rojo e instanciar $U^{*}$ es lo que hace que $b$ sea rojo, entonces U y $U^{*}$ son "rojeces" indiscernibles. Esta caracterización de los universales indiscernibles no es una definición de ellos -en particular, uno no debería explicar la indiscernibilidad de dos universales indiscernibles en términos de la similitud exacta en un cierto respecto de los particulares que los instancian, ya que una similitud exacta tal en un respecto debe ser explicada en términos de los universales que esos particulares instancian.

Indiscernibilidad y similitud perfecta son a menudo asociados con compartir todas las propiedades. Pero hay un sentido en el cual los universales indiscernibles, como los he caracterizado, no necesitan compartir todas sus propiedades. Por ejemplo, si U y U* son dos "rojeces" perfectamente similares, y U está instanciado por el particular $a$, mientras que $\mathrm{U}^{*}$ está instanciado por el particular $b, \mathrm{U}$ y U* no compartirán, entre otras cosas, la propiedad de ser instanciado por $b$.

De hecho, sin importar cómo sean ellos caracterizados, los universales numéricamente diferentes pero indiscernibles nunca compartirán todas sus propiedades: $U$ y $U^{*}$ nunca 
compartirán la propiedad de ser idéntico a $U$. Pero normalmente cuando la gente asocia la indiscernibilidad con el compartir todas las propiedades, hay una restricción implícita a cierto tipo de propiedad; por ejemplo, propiedades intrínsecas o puramente cualitativas. Pero nada de lo que he dicho en mi caracterización de los universales indiscernibles implica que ellos deban tener todas sus propiedades intrínsecas o puramente cualitativas en común; pues todo lo que he dicho es que los universales indiscernibles son aquellos universales que confieren similitud perfecta en un respecto a los particulares que los instancian. ¿Significa esto que mi caracterización de los universales indiscernibles es defectuosa? No, porque no es una posición incoherente pensar que los universales carecen de cualquier naturaleza intrínseca o cualitativa pero al mismo tiempo confieren un carácter cualitativo a los particulares que los instancian. De hecho, no es implausible que la rojez universal no sea ella misma roja - pero si no es roja, es plausible que no tenga una naturaleza cualitativa en absoluto. Podría argüirse que incluso si los universales no tienen una naturaleza cualitativa, ellos deben tener algunas propiedades generales intrínsecas, como ser un universal, o ser una entidad. Pero si esto no nos da lo que uno necesita, porque o bien propiedades como éstas son compartidas por todos los universales, en cuyo caso todos los universales serían indiscernibles, o bien ellas son compartidas por muchos universales que confieren un carácter cualitativo diferente a sus instancias, en cuyo caso algunos universales indiscernibles tendrían instancias que no se asemejan perfectamente en ningún respecto

Pero si los universales tienen una naturaleza cualitativa, deberíamos considerar que los universales indiscernibles son aquellos que comparten su naturaleza cualitativa, el cual es un resultado que tiene mi caracterización de los universales indiscernibles. Porque si los universales tienen una naturaleza cualitativa, el carácter cualitativo que ellos le confieren a sus instancias depende de esa naturaleza. Por tanto, si los universales tienen una naturaleza cualitativa, los universales que confieren similitud perfecta en un respecto a los particulares que los instancian son universales que comparten su naturaleza cualitativa. De este modo, mi caracterización de los universales indiscernibles es una condición que debe ser satisfecha por ellos, sin importar si los universales tienen o no una naturaleza cualitativa.

Argumentaré que los universales indiscernibles pueden realizar un trabajo filosófico útil, y mis argumentos para esta tesis no presupondrán ni que los universales tienen una naturaleza cualitativa ni que no la tienen. Por tanto, mi conclusión será aplicable a concepciones de los universales en las cuales ellos tienen una naturaleza cualitativa y concepciones en las cuales ellos no la tienen.

Armstrong no cree en universales indiscernibles. Por ejemplo, ha dicho que "universales diferentes no pueden asemejarse con exactitud, porque si lo hicieran, entonces ellos serían el mismo universal" (1997, 50). Armstrong no está solo en esto. John Heil, por ejemplo, ha dicho que "[e]n tanto que analicemos las propiedades, la similitud exacta entre las propiedades equivale a identidad" (2003, 154). David Lewis, quien no creía 
en universales, brevemente los consideró - los llamó anfibios- como una manera de dar sentido a los universales estructurales. Pero él pensó que no estaba claro que ellos fueran realmente universales, de acuerdo con el rol teorético que se supone que los universales juegan (cf. Lewis 1999, 98). (Consideraré la objeción de Lewis a los universales indiscernibles en la Sección 4). De hecho, según tengo entendido, todos los filósofos que creen en los universales creen que no puede haber universales indiscernibles.

Y de acuerdo con algunos filósofos, como John Wisdom, D. C. Williams y Douglas Ehring, lo que distingue a los universales de los particulares es precisamente que los primeros no pueden ser indiscernibles, mientras que los últimos pueden (un criterio tal de distinción entre particulares y universales será sometido a discusión en la Sección 4).

En algunas concepciones de los universales, es plausible que no haya universales indiscernibles. Por ejemplo, Peter van Inwagen tiene una concepción de las propiedades, o universales, como afirmables (cf. 2004, 27-29). Desde este punto de vista, una propiedad es lo que puede ser afirmado (asserted) de algo. No le puedo encontrar mucho sentido a la idea de que hayan dos afirmables (assertibles) perfectamente similares, ya que no le puedo encontrar mucho sentido a un afirmable que confiera carácter cualitativo a un particular.

Ya sea que esté o no en lo correcto acerca de los afirmables, no hay nada en la concepción de los universales como entidades que son idénticas a través de sus instancias que excluya la posibilidad de los universales indiscernibles. De hecho, no hay nada en esta concepción que excluya la posibilidad de que diferentes universales tengan la misma naturaleza cualitativa, $y$, por tanto, que confieran similitud perfecta en un respecto a los particulares que los instancian, y nada en esta concepción excluye la posibilidad de diferentes universales sin una naturaleza cualitativa, pero que, sin embargo, confieran similitud perfecta en un respecto a los particulares que los instancian. No es ninguna sorpresa, entonces, que Armstrong no dé ningún argumento cuando dice que universales diferentes no pueden asemejarse con exactitud, porque si lo hicieran, entonces, ellos serían el mismo universal -no creo que un argumento tal exista.

Pero que los universales indiscernibles son posibles o concebibles no quiere decir que uno debería postularlos. Sin embargo, en este artículo sostendré que los universales indiscernibles pueden hacer un trabajo útil y que, de este modo, podría haber razones para postular universales indiscernibles. Por consiguiente, los universales indiscernibles merecen mayor consideración y examen. En la Sección 2, argumentaré que postular los universales indiscernibles puede permitir que una teoría de los universales identifique los particulares con bundles de universales. En la Sección 3, argumentaré que postular los universales indiscernibles puede permitir una teoría de los universales que desarrolle una explicación de la semejanza de universales cuantitativos que evite las objeciones a las que la explicación de Armstrong se enfrenta. Finalmente, en la Sección 4 responderé 
a algunas objeciones y objetaré el criterio de acuerdo con el cual la distinción entre particulares y universales yace en que mientras puede haber particulares indiscernibles, no puede haber universales indiscernibles.

\section{Los universales indiscernibles y la Bundle Theory}

Tradicionalmente, se ha pensado que la Bundle Theory de Universales implica la Identidad de los Indiscernibles para particulares (cf. Armstrong 1978a, 91; Loux 1998, 107). De acuerdo con la versión relevante de la Identidad de los Indiscernibles, no puede haber particulares numéricamente distintos pero perfectamente similares. La Bundle Theory dice que los particulares están completamente constituidos por universales. Pero ya que los universales son lo que confiere similaridad a los particulares, y que se supone que los universales son idénticos en sus instancias, se concluye que si los particulares son completamente constituidos por universales, los particulares numéricamente distintos no pueden ser perfectamente similares. Tal es el argumento según el cual la Bundle Theory implica la Identidad de los Indiscernibles.

Pero si esto es así, esto es un problema para la Bundle Theory, porque se considera que la Identidad de los Indiscernibles es falsa para los particulares. Se considera que es falsa, ya que parece que podría haber habido particulares perfectamente similares, por ejemplo, un mundo habitado solamente por dos esferas de hierro con el mismo diámetro, el mismo color, la misma masa, la misma temperatura, etc. (cf. Black 1952, 156).

No obstante, como argumentaré en esta sección, una cosa importante que los universales indiscernibles pueden hacer es permitir que la Bundle Theory de Universales evite el compromiso con la identidad de particulares indiscernibles.

Ahora bien, he argumentado que la Bundle Theory no implica por sí misma la Identidad de los Indiscernibles (cf. Rodriguez-Pereyra 2004), pues el argumento de que la Bundle Theory implica la Identidad de los Indiscernibles depende de la suposición de que dos particulares no pueden tener exactamente los mismos constituyentes, y esa es una suposición a favor de la cual no hay razón alguna. Así, al rechazar esa suposición, la Bundle Theory se vuelve consistente con particulares numéricamente distintos, pero perfectamente similares. Cuando hay particulares numéricamente distintos pero perfectamente similares, hay particulares numéricamente distintos que tienen exactamente los mismos constituyentes: dos particulares, un bundle de universales.

Esto requiere distinguir los particulares de los bundles. Porque si los particulares fueran idénticos a los bundles de universales, no podría haber un bundle cuando hay dos particulares. Entonces, ¿qué son los particulares? Se ha propuesto una versión de la Bundle Theory de acuerdo con la cual hay bundles, y hay instancias de bundles, y los particulares 
son instancias de los bundles, de tal manera que cuando hay particulares numéricamente distintos pero perfectamente similares, hay un bundle de universales y dos instancias de éste (cf. Rodriguez-Pereyra 2004, 78).

Pienso que ésta es una buena versión de la Bundle Theory. Pero esta teoría tiene un costo, a saber, que los particulares no pueden ser identificados con bundles de universales. Se obtendría una simplificación si la identificación de los particulares con los bundles pudiera recuperarse, manteniendo al mismo tiempo la Bundle Theory separada de la Identidad de los Indiscernibles. ¿Puede ser esto llevado a cabo? Sí, con la ayuda de los universales indiscernibles. Permítanme explicar.

Hay otro supuesto más en el argumento de que la Bundle Theory implica la identidad de los indiscernibles, a saber, que los universales instanciados por dos particulares perfectamente similares deben ser numéricamente idénticos. Presumiblemente, la razón detrás de este supuesto es que sólo el compartir numéricamente el mismo universal puede conferir semejanza a los particulares. Pero los universales indiscernibles confieren tanta semejanza a los particulares como lo hace un solo universal instanciado por ellos. Esto es, dos particulares, uno de los cuales instancia un universal $U$ y otro de los cuales instancia un universal indiscernible $U^{*}$, se asemejarían entre sí en el sentido relevante, tanto como dos particulares que instancien U -esto es, dos particulares, uno de los cuales instancia un universal $U$ y otro de los cuales instancia un universal indiscernible $U^{*}$, se asemejarían entre sí exactamente en el sentido relevante. De esta manera, si hay universales indiscernibles, dos particulares podrían ser perfectamente similares no porque instancien numéricamente los mismos universales sino porque instancian universales indiscernibles, esto es, universales numéricamente distintos pero perfectamente similares.

Por consiguiente, al postular universales indiscernibles y rechazar el supuesto de que los universales instanciados por dos particulares perfectamente similares deben ser numéricamente idénticos, la Bundle Theory puede sostener que hay particulares indiscernibles. Aún más, puede hacerlo al mismo tiempo que mantiene la identificación de los particulares con bundles de universales. Supóngase que los particulares $a$ y $b$ son indiscernibles. En tal caso ellos instancian universales indiscernibles. Y mientras que el particular $a$ es el bundle constituido por los universales $\mathrm{U}, \mathrm{V}, \mathrm{W}, \ldots$, el particular $b$ es el bundle constituido por los universales $U^{*}, V^{*}, W^{*}, \ldots$ Esto es sólo un ejemplo y nada aquí implica que dos particulares indiscernibles no puedan instanciar uno y el mismo universal. Todo lo que se requiere es que los particulares en cuestión instancien al menos un par de universales indiscernibles; por consiguiente, el particular a podría ser el bundle constituido por los universales $\mathrm{U}, \mathrm{V}, \mathrm{W}, \ldots$, y el particular $b$ podría ser el bundle constituido por los universales $\mathrm{U}^{*}, \mathrm{~V}, \mathrm{~W} \ldots$

Esta no es la única manera en la cual uno puede identificar los particulares con bundles sin comprometerse con la Identidad de los Indiscernibles. Porque uno podría mantener que los particulares son idénticos a los bundles, pero bundles distintos pueden tener exac- 
tamente los mismos universales como constituyentes ${ }^{1}$. Qué teoría es preferible depende de si se prefiere universales indiscernibles o distintos bundles de universales constituidos por exactamente los mismos universales. Mi punto aquí no es argumentar que los universales indiscernibles son esenciales para volver consistente la Bundle Theory con el rechazo de la Identidad de los Indiscernibles, sino sólo mostrar que ellos proveen una manera de hacerlo, y que tendrá que argumentarse que no es la mejor manera.

\section{Los universales indiscernibles y la semejanza entre universales cuantitativos}

El segundo trabajo que los universales indiscernibles pueden hacer para una teoría de los universales es proporcionar una mejor explicación de la semejanza entre los universales cuantitativos que la teoría de Armstrong, la teoría más elaborada de semejanza entre universales. Sin embargo, no está claro que su explicación funcione para todos los universales, por ejemplo, los colores. Y, de hecho, tengo serias dudas de que funcione en ese caso. Pero esto no es importante en sí mismo, ya que no hay razón por la cual la explicación de la semejanza entre universales debiera aplicarse a los universales de todos los tipos. Quizás explicaciones diferentes funcionen para diferentes tipos de universales. La explicación de Armstrong funciona razonablemente bien para los universales cuantitativos como masas, longitudes y duraciones. Sin embargo, incluso en estos casos la explicación enfrenta algunos problemas. Argumentaré que una explicación de la semejanza entre universales cuantitativos como masas, longitudes y duraciones, basada en los universales indiscernibles, elude algunos de los problemas enfrentados por la explicación de Armstrong (de modo que, de ahora en adelante, cuando en esta sección hable de universales sin calificación, debe entenderse que me refiero a universales cuantitativos como masas, longitudes y duraciones). Me voy a concentrar en la explicación de Armstrong para las masas, ya que es aquí donde se puede ver con mayor claridad las dificultades que enfrenta. Primero, voy a describir la explicación de Armstrong y las dificultades que ella enfrenta, y luego voy a argumentar que una cierta explicación basada en los universales indiscernibles puede eludir dichas dificultades.

De acuerdo con Armstrong, cada par de universales que se asemejan son parcialmente idénticos, de manera que cada par de masas son parcialmente idénticas (cf. 1978b, 123; 1997, 51). Esto significa que para dos masas cualesquiera, una contendrá a la otra como una parte. Considérese la masa de 3 kilogramos y la masa de 2 kilogramos. La masa de 2 kilogramos es una parte de la masa de 3 kilogramos. Ellas también tienen otras partes en común, pues la masa de 1 kilogramo es parte tanto de la masa de 2 kilogramos y la masa de 3 kilogramos.

¿Qué es para un universal ser parte de otro? De acuerdo con Armstrong, que un universal sea parte de otro universal significa que siempre que un particular instancie el último,

\footnotetext{
${ }^{1}$ Estoy en deuda con Nick Jones en este punto.
} 
una parte del particular instancia el primero (cf. 1997, 55). Por consiguiente, siempre que algo tenga una masa de tres kilogramos, una parte de esa cosa es de dos kilogramos de masa, y otra parte es de un kilogramo de masa y así sucesivamente, para todas las masas menores que la masa de 3-kilogramos.

Pero la explicación no es perfecta incluso en el caso de las masas. Veamos algunos de los problemas que enfrenta. En primer lugar, parece que hay partículas del tamaño de un punto, como los electrones. Los electrones tienen masa. La masa de los electrones tiene otras masas como partes. Pero los electrones no tienen partes. Pero, de acuerdo con Armstrong, si algo instancia una masa, debe tener partes que instancien las partes de la masa.

Armstrong ofrece una solución a este problema. Él señala que donde hay una partícula del tamaño de un punto, ahí existen muchos otros particulares, tal vez infinitamente muchos, instanciado las partes de la masa instanciad por tal partícula (cf. 1997, 65). Así, existen muchos, tal vez infinitamente muchos, particulares localizados exactamente en el mismo punto, y ellos son las partes de tal partícula. Pero esta es una conclusión extraña (y una que está en tensión con su propia metodología para la metafísica).

Más aun, nótese que esta estrategia para partículas del tamaño de un punto no se aplicaría a objetos simples, que por definición no tienen partes. Pero la razón por la cual Armstrong implementa esta estrategia en el caso de las partículas del tamaño de un punto es que, en su teoría, cualquier cosa que instanciara un universal complejo debe tener partes que instancien las partes de tal universal. Esto significa que en su teoría ningún simple puede instanciar un universal complejo, y que por tanto debe excluir, por ejemplo, objetos simples extendidos.

Maya Eddon ha señalado que existen otros problemas con la teoría de Armstrong para partículas del tamaño de un punto. Primero, esta teoría vuelve metafísicamente imposible que exista un solo objeto que instancie masa alguna y que ocupe un punto en un cierto instante de tiempo. Pero esto pareciera ser metafísicamente posible y es plausible que de hecho sea el caso (cf. Eddon 2007, 391-2).

Segundo, existe una falta de consistencia entre el tratamiento que Armstrong hace de los universales cuantitativos instanciados por partículas del tamaño de un punto y los instanciados por objetos extendidos. De acuerdo con Armstrong, toda partícula del tamaño de un punto que instancia un universal cuantitativo tiene el mismo patrón de partes que tendría si se extendiese. Pero entonces un científico debería ser capaz de aislar las partes de partículas del tamaño de un punto de la misma manera como lo podría hacer con las partes un objeto extendido. Sin embargo, no existe evidencia científica que existan partículas con la mitad de la masa de un electrón (cf. Eddon 2007, 392).

Tercero, no existe una justificación independiente para la suposición de Armstrong que toda partícula del tamaño de un punto tiene partes. Así, aunque es plausible postular partes propias para un objeto con masa si tal objeto tiene extensión, no lo es para partí- 
culas del tamaño de un punto. Pues incluso antes de adoptar la teoría de Armstrong, ya teníamos razón para creer en tales partes para objetos extendidos. Pero la única razón que tenemos para postular partes para partículas del tamaño de un punto es que son requeridas por la teoría de Armstrong (cf. Eddon 2007, 392).

Ahora, los problemas para la teoría de Armstrong no se limitan a las implicancias que tiene para partículas del tamaño de un punto o simples que se extienden. Considérese las cantidades intensivas, esto es, cantidades tales que las partes de la entidad que posee la cantidad en cuestión poseen la misma cantidad. La temperatura y la dureza son ejemplos de tales cantidades. Estas propiedades presentan un problema para Armstrong. Consideremos un particular cualquiera que posee una cantidad intensiva. Sus partes debieran instanciar cantidades menores a la que posee, pero esto no es necesariamente así. De este modo, Armstrong excluye las cantidades intensivas de su metafísica (cf. 1997, 94). Pero éste es un costo para su teoría y la vuelve rehén de la posibilidad que se encuentren cantidades intensivas irreductibles.

Los universales indiscernibles pueden explicar la semejanza entre universales cuantitativos de manera de evitar los problemas de la teoría de Armstrong. Los problemas para Armstrong surgen del hecho que si un universal es parte de otro, entonces las partes del particular que instancian el segundo universal deben instanciar el primer universal. Pero en algunos casos, los particulares en cuestión no tendrán partes (electrones, simples extendidos) y en otros casos las partes del particular no instancian las partes del universal (cantidades intensivas).

La solución a estos problemas es disociar la idea que los universales tienen partes de la idea que las partes de los particulares que instancian un universal instancian sus partes. Esto se puede lograr sin dejar de sostener que masas más grandes contienen a masas más pequeñas. Así, incluso si las partes de un particular que instancia a un universal no instancian necesariamente las partes de tal universal, seguiría siendo el caso que el universal de 5 kilogramos tiene como parte un universal de 4 kilogramos.

Consideremos primero las cantidades extensivas. Si un particular tiene partes, y tiene una cierta masa, las masas que corresponden a las partes en cualquier partición de ese objeto sumarán la masa del particular en cuestión. Por ejemplo, si un particular pesa 5 kilogramos, la suma de las masas de las partes que corresponden a cualquier partición sumarán 5 kilogramos. Algunas particiones consistirán de cinco partes de 1 kilogramo; algunas de dos partes de 2 kilogramos y una de un 1 kilogramo; algunas particiones de una parte de 2 kilogramos y otra de 3 kilogramos; y algunas particiones de una parte de 4 kilogramos y una de 1 kilogramo (para simplificar ignoro cualquier partición que incluya partes con masas que no sean medibles por números enteros). Ninguna partición incluirá más de cinco partes de 1 kilogramo, ninguna partición incluirá más de dos partes de 2 kilogramos, ninguna partición incluirá más de una parte de 3 kilogramos y ninguna partición incluirá más de una parte de 4 kilogramos. 
Una sugerencia natural, a ser desarrollada aquí, es que la estructura mereológica de un universal corresponde a este tipo de estructura. Esto es, un universal de 5 kilogramos está compuesto por un universal de 4 kilogramos, un universal de 3 kilogramos, dos universales indiscernibles de 2 kilogramos y cinco universales indiscernibles de 1 kilogramo. En general, un universal que corresponde a una cantidad extensiva está compuesto de universales que serían instanciados por las partes del particular que lo instancia, si tuviera partes y todas sus partes tuvieran partes; y está compuesto de tantos universales de un tipo como partes pudieran existir e instancian universales de ese tipo en cualquier partición del particular en cuestión. De este modo, un universal de 4 kilogramos está compuesto de un universal de 3 kilogramos, dos universales indiscernibles de 2 kilogramos y cuatro universales indiscernibles de 1 kilogramo.

Esta teoría realiza una afirmación adicional: las cantidades intensivas como la temperatura y la dureza también tienen este tipo de estructura. Así, un universal que corresponde a una cierta temperatura está compuesto de universales que serían instanciados por las partes del particular que instancia a tal universal si la temperatura en cuestión fuese extensiva. Así, un universal, de 5 kelvins está compuesto de un universal de 4 kelvins, un universal de 3 kelvins, dos universales indiscernibles de 2 kelvins y cinco universales indiscernibles de 1 kelvin. ${ }^{2}$ Una versión más extrema de la teoría sostiene que todas las cantidades o incluso todo universal complejo tiene esta estructura. Pero limitaré mi atención aquí a la versión más débil de la teoría que sostiene que sólo las cantidades como masas, longitudes, duraciones y temperaturas tienen la estructura en cuestión. ¿Por qué no considerar la teoría que sostiene que todas las cantidades tienen la estructura en cuestión? Porque no todas ellas parecen tener tal estructura. Las cargas, por ejemplo, tienen valores positivos y negativos y pareciera que no existe una unidad de carga que sea un componente de cualquier otra carga. Aunque tal vez lo pudiera haber. Tal vez toda carga está compuesta, entre otras cosas, por una carga de -e/3 y una carga de e/3, de manera que estas dos cargas se cancelarían mutuamente. Sin embargo, no puedo desarrollar y explorar aquí tal hipótesis especulativa. Ni tampoco lo necesito, puesto que como dije antes, no hay razón para que sólo una teoría de la semejanza entre universales deba funcionar para todo universal. ${ }^{3}$

Ahora, la relación de parte es transitiva, de modo que los cuatro universales indiscernibles de 1 kilogramo que son partes de un universal de 4 kilogramos deben ser partes de un universal de 5 kilogramos del cual el universal de 4 kilogramos es parte. Pero el uni-

\footnotetext{
${ }^{2}$ Uso la escala Kelvin en vez de la escala Celsius o Fahrenheit porque en la escala Kelvin el punto cero es, por definición, el cero absoluto. Estoy en deuda con Huw Price en este punto.

${ }^{3}$ Estoy en deuda con Markku Keinänen por el punto que las cargas no tienen la estructura que propongo para las masas, longitudes, duraciones y temperaturas.
} 
versal de 5 kilogramos tiene 5 universales de 1 kilogramo como partes — ¿cuáles cuatro de estas cinco partes son partes del universal de 4 kilogramos? Este será un hecho bruto y arbitrario, del tipo que cualquier teoría que postule indiscernibles deberá aceptar.

Una afirmación crucial que la teoría hace es la siguiente: cuando un particular instancia un universal, instancia sus partes también. Así, un particular de 5 kilogramos instancia el universal de 4 kilogramos, el universal de 3 kilogramos, los dos universales indiscernibles de 2 kilogramos y los cinco universales indiscernibles de 1 kilogramo que son partes del universal de 5 kilogramos que instancia. Esta afirmación es crucial porque respeta la plausible idea que cuando un universal es instanciado, sus partes son instanciadas también, sin que sea necesario que las partes del universal sean instanciadas por las partes del particular que lo instancia. En efecto, esta afirmación se apega estrictamente más a la idea que un universal inmanente, en su totalidad, está presente donde y cuando sea instanciado que la idea alternativa que las partes de un universal son solo instanciadas por las partes de los particulares que lo instancian.

Pero decir que un particular que instancia un universal instancia sus partes presenta una dificultad. Pues la afirmación parece suponer que todos los objetos tienen masas infinitamente grandes. Por ejemplo, consideremos un particular de 2 kilogramos. Este particular instanciaría dos universales de 1 kilogramo que son partes del universal de 2 kilogramos que instancia. Pero si instancia estos otros dos universales, cada uno de ellos debería proporcionarle al particular una masa de 1 kilogramo. Pero entonces su masa total es de cuatro kilogramos $(2+1+1)$. Pero si es así, el particular en cuestión debería también instanciar un universal de 4 kilogramos. Y si es así, el particular en cuestión debería también instanciar un universal de 3 kilogramos, los dos universales de 2 kilogramos y los cuatro universales de 1 kilogramo que son partes del universal de 4 kilogramos que instancia. Estos universales deben conferir una masa al particular. Pero entonces su masa total (dado que ya hemos contado un universal de 2 kilogramos y dos de 1 kilogramo) es de 11 kilogramos $(4+3+2+1+1)$. Pero entonces instancia un universal de 11 kilogramos. Y así sucesivamente. Este argumento se generaliza, de modo que todo particular con una cierta masa es infinitamente "masivo".

Claramente, uno debe rechazar esta conclusión. El argumento presupone que las masas son aditivas. Pero no veo un gran prospecto en rechazar tal suposición. Y no nos servirá afirmar que la masa de un particular corresponde a la masa del universal más grande que instancia. Pues, ¿tienen los particulares una masa más grande? De acuerdo con el argumento previo, los particulares no tienen una masa más grande.

El argumento presupone que si un particular instancia una masa de $n$ kilogramos, entonces $n$ kilogramos es una masa del particular. Esto se puede justificar apelando al principio general que si algo instancia un universal $\mathrm{F}$, entonces esa cosa es $\mathrm{F}$ - esto es, el principio que instanciar $\mathrm{F}$ es una condición suficiente para ser F. 
Podría parecer que rechazar este principio general es algo negativo porque significaría cortar la conexión entre instanciar F y ser F. Pero la conexión no necesita ser cortada - tal conexión se podría entender de una manera diferente. Así, un defensor de la teoría que estamos considerando debería rechazar el principio que instanciar $\mathrm{F}$ es una condijo suficiente para ser $\mathrm{F}$ y reemplazarlo por la siguiente condición suficiente: un particular es $\mathrm{F}$ si instancia $F$ y no instancia ningún universal $G$ tal que (a) $G$ es una determinación del mismo determinable que F y (b) F es una parte propia de G.

Con esta nueva condición suficiente en su lugar, un particular de 2 kilogramos que instancia un universal de 1 kilogramo no es por tanto también un particular de 1 kilogramo y así el argumento que los particulares con masa son infinitamente "masivos" no puede siquiera formularse. Más aun, la nueva condición suficiente explica por qué un particular de 2 kilogramos que instancia un universal de 2 kilogramos y no otro universal de masa más grande pesa dos kilogramos, i.e., explica por qué un particular de 2 kilogramos que instancia un universal de 2 kilogramos y no otro universal de masa más grande es un particular de dos kilogramos. También es cierto que algo es F si instancia F y no instancia ningún universal de cual $\mathrm{F}$ es una parte propia. Pero esto no sería explicar por qué un particular que instancia un universal de 2 kilogramos y no otro universal de masa más grande pesa dos kilogramos, i.e., por qué tal particular es un particular de 2 kilogramos. Pues el particular de 2 kilogramos, si es rojo, también instancia el universal conjuntivo ser de 2 kilogramos y rojo, del cual el universal de 2 kilogramos es una parte

¿Qué universales son instanciados por las partes de un particular? Depende de si acaso el universal en cuestión es una cantidad intensiva o extensiva. Si un universal es una cantidad extensiva, las partes del particular que lo instancia instancian sus partes. Consideremos un particular de 5 kilogramos con partes. Instanciará cinco universales indiscernibles de 1 kilogramo. ¿Serán los universales de 1 kilogramo instanciados por sus partes universales que son instanciados por el particular mismo? La teoría se simplifica si asumimos que en efecto esto es el caso.

Pero nótese que esto sólo quiere decir que los universales instanciados por las partes de un particular son partes de los universales instanciados por el particular. No significa entonces que cualquier parte de un universal instanciado por el particular en cuestión es instanciado por una de las partes del particular. Pues considérese dos particulares separados de 5 kilogramos que instancian exactamente el mismo universal de 5 kilogramos. Imaginemos que vienen a componer un nuevo compuesto particular de 10 kilogramos. Es plausible pensar que componer un nuevo objeto no hace que los objetos componentes cambien en sus universales, especialmente si la composición se logra, digamos, por llegar a estar en contacto. Pero el nuevo particular compuesto instanciará un universal de 10 kilogramos que tendrá como partes dos universales indiscernibles de 5 kilogramos. Solo 
uno de estos dos universales de 5 kilogramos será instanciado por la partes del particular de 10 kilogramos. ${ }^{4}$ Pero ambos universales serán instanciados por el particular de 10 kilogramos.

Como es claro a partir del ejemplo anterior, cosas separadas, esto es, cosas que no comparten partes, pueden instanciar el mismo universal en esta concepción. Nada excluye la posibilidad que el mismo universal de 5 kilogramos sea instanciado por dos particulares diferentes sin ninguna relación mereológica entre ellos. Pero si esto ocurriera, los universales de 1 kilogramo instanciados por las partes de los particulares de 5 kilogramos serán universales de 1 kilogramo que son partes del universal de 5 kilogramos en cuestión. Y si uno de estos particulares de 5 kilogramos es una parte de un particular de 10 kilogramos, el particular de 10 kilogramos instanciará el universal de 10 kilogramos del cual ese universal de 5 kilogramos es parte.

Ahora, si un universal es una cantidad intensiva, las partes del particular que lo instancia pueden también instanciarlo. En este caso, el universal y todas sus partes serán instanciadas tanto por el particular como por sus partes.

Existe otra manera en la cual esta teoría diverge de la de Armstrong. Para Armstrong, la semejanza entre universales es un asunto de identidad parcial en el sentido que, de dos universales que se asemejan, uno debe ser parte del otro. Esto no puede ser el caso para nuestra teoría. Pues ninguno de los dos universales indiscernibles es parte del otro. Pero la conexión entre semejanza para universales y la noción de identidad parcial puede preservarse de otro modo. Uno debería decir que para que dos universales se asemejen imperfectamente o bien uno de ellos es parte del otro, o una parte de uno de ellos es indiscernible de una parte del otro (puesto que la indiscernibilidad es reflexiva, podemos simplificar las cosas estipulando que para que dos universales se asemejen imperfectamente uno de ellos debe ser indiscernible de una parte del otro). Así, un universal de 2 kilogramos y un universal de 1 kilogramo se asemejan entre sí porque el segundo es parte del primero. Del mismo modo, un universal de 1 kilogramo y un universal de 2 kilogramos tal que el primero no es el parte del segundo se asemejaran entre sí porque el universal de 1 kilogramo es indiscernible de una parte del universal de 2 kilogramos.

La explicación previa de la semejanza imperfecta entre universales presupone la noción de semejanza perfecta entre universales, dado que la explicación se formula en términos de universales indiscernibles, y los universales indiscernibles son universales perfectamente semejantes. Pero es obvio que en esta teoría, la indiscernibilidad o la semejanza perfecta entre universales no puede explicarse en términos de identidad completa. Pues en esta teoría existen universales indiscernibles que no son numéricamente idénticos. De esta manera, los universales son perfectamente similares cuando son numéricamente idénticos o bien indiscernibles.

\footnotetext{
${ }^{4}$ Le debo este ejemplo a Martin Pickup.
} 
Este es un esbozo grueso de cómo una teoría que postula universales indiscernibles explicaría la semejanza de universales cuantitativos. El punto importante aquí es que ni los electrones, ni las entidades simples, ni las cantidades intensivas presentan un problema de suyo para esta teoría, pues esta teoría no requiere que las partes de un universal sean instanciadas por las del particular que lo instancia, ni requiere que un particular que instancia un universal tenga partes.

Consideremos un electrón de masa $m$. Esa masa se asemeja a masas más pequeñas incluso si el electrón en cuestión no tiene partes. Esto ocurre porque o bien las masas más pequeñas son partes de $m$ o bien porque son indiscernibles de partes de $m$. Y esto no requiere que las masas más pequeñas sean instanciadas por entidades diferentes al electrón en cuestión, pues el electrón mismo instanciará las partes de $m$. Exactamente las mismas consideraciones son válidas para los casos de otros objetos simples.

La teoría tampoco enfrenta las objeciones de Eddon a la teoría de Armstrong. Primero, en la teoría recién presentada es metafísicamente posible que exista un objeto que instancie una masa y que ocupe un punto en un instante de tiempo dado, y también es metafísicamente posible que exista un objeto simple extendido con masa.

Segundo, puesto que la nueva teoría no postula partículas que tengan la mitad de la masa de un electrón, no sería una objeción para ella que la ciencia no haya encontrado tales partículas. Se podría decir que la ciencia aún no ha encontrado los universales indiscernibles que postula esta nueva teoría, pero dados los problemas de la teoría de Armstrong, la evidencia disponible a favor de partículas del tamaño de un punto u otros objetos simples es evidencia también a favor de la teoría que presento aquí.

Tercero, puesto que la nueva teoría no postula partes de partículas del tamaño de un punto, no hay problema en tener una justificación independiente para tal postulado. Se podría argumentar que la nueva teoría no tiene una justificación independiente para postular universales indiscernibles como partes de otros universales. En efecto, tal justificación no existe. Pero este es un aspecto tan básico de la teoría que no requiere una justificación independiente. Este es un aspecto es definitorio de la nueva teoría.

De la misma manera, las cantidades intensivas como la temperatura no son un problema tampoco. Consideremos dos particulares, uno que instancia una temperatura $q$ y otro que instancia una temperatura menor $q^{*}$. Estas temperaturas se asemejan entre sí porque o bien $q^{*}$ es parte de $q$ o bien es indiscernible de una parte de $q$. Y no es un requisito de la teoría que alguna parte del particular que instancia $q$ instancie $q^{*}$.

\section{Objeciones y respuestas}

Otro uso para los universales indiscernibles es que nos ayudan a dar cuenta de universales estructurales. Esto es algo que vislumbró David Lewis (cf. 1999, 98-100) por lo que no me adentraré en ello aquí. En esta sección me ocuparé de algunas objeciones a la 
teoría antes descrita. La primera objeción es que los universales indiscernibles no son necesarios para dar cuenta de la semejanza entre universales. Pues el objetor dice que todo el trabajo está ya hecho por el requerimiento de que las partes de los universales estén instanciadas por el particular que instancia el universal más que por sus partes mismas, por lo que no sería necesario exigir que algunas de las partes del universal sean universales indiscernibles.

Pero, asumiendo algunas suposiciones plausibles, se puede mostrar que si las cantidades más grandes tienen cantidades más pequeñas como partes, éstas tienen cantidades indiscernibles como partes. Si se asume que las cantidades son universales, de esto resulta que los universales cuantitativos tienen universales indiscernibles como partes. Asúmase que un cierto universal de 4 kilogramos es parte de un cierto universal de 5 kilogramos. Según el Principio de Suplementación Débil, debe haber una parte del universal de 5 kilogramos que está separado del universal de 4 kilogramos. En aras del ejemplo, asúmase que tal parte es un universal de 1 kilogramo, y llámese a tal universal de 1 kilogramo $a$. Pero el universal de 4 kilogramos debe tener un universal de 1 kilogramo como una de sus partes. Sea $b$ el universal de 1 kilogramo que es parte del universal de 4 kilogramos. En la medida que $a$ está separado del universal de 4 kilogramos y pero $b$ no lo está, $a$ y $b$ deben ser universales de 1 kilogramo numéricamente distintos. Pero es plausible que universales de 1 kilogramo que son numéricamente distintos sean universales indiscernibles y, así, es plausible que hayan universales indiscernibles. Además, también es plausible que la relación de parte sea transitiva. Por lo tanto, los dos universales de 1 kilogramo son partes del universal de 5 kilogramos original. Generalizando este argumento, se concluye que si las cantidades más grandes tienen cantidades más pequeñas como partes, las cantidades más grandes tienen universales indiscernibles como partes.

Sin duda, uno podría rechazar las suposiciones que el Principio de Suplementación Débil es verdadero, que las cantidades numéricamente distintas del mismo valor son indiscernibles y que la relación de parte es transitiva. Por ejemplo, Maureen Donnelly (cf. 2011) ha presentado razones para dudar de la validez general del Principio de Suplementación Débil, si bien no es claro que sus razones para dudar del Principio apliquen al caso de los universales de masa. En cualquier caso, mi argumento puede también funcionar con su principio $\mathrm{MA}_{3}$ más débil, de acuerdo al cual si $x$ es una parte de $y$, pero $y$ no es parte de $x$, entonces existe un $z$ que es parte de $y$ pero $y$ no es parte de $z$, tal que $z$ y $x$ están separados (cf. 2011, 234). Esto dado que una masa más grande y una más pequeña son partes mutuas. Sin embargo, lo único que me interesa aquí es establecer que estas suposiciones son plausibles, no indubitables.

Una segunda objeción es que una teoría que postula universales indiscernibles es inconsistente con los compromisos básicos del realismo acerca de los universales. Por ejemplo, Ehring (cf. 2011, 37-8) sugiere que una teoría que está comprometida con los universales indiscernibles es inconsistente con un principio fundamental del realismo según el cual los objetos que no comparten universales no son ni similares ni exactamente 
similares. Tal parece ser la idea que Adam Pautz tiene en mente cuando escribe: "Si universales diferentes pueden asemejarse exactamente, entonces dos particulares podrían asemejarse exactamente y aun así instanciar diferentes universales (no relacionales), lo cual es contrario a la teoría de universales" (Pautz 1997, 110, nota 6). Esta es también la objeción de Lewis para los universales indiscernibles (cf. Lewis 1999, 98). La teoría que Ehring tiene en mente es lo que él llama Primitivismo Categorial, esto es, la postura que los universales simples de la misma adicidad (adicity) son exactamente similares pero numéricamente diferentes. Esa no es la postura que se explora en este artículo, en la medida que la postura que se explora aquí no se restringe a universales simples, ni implica que todos los universales con la misma adicidad sean indiscernibles. Sin embargo, la postura asumida aquí sí es inconsistente con lo que Ehring considera como un principio fundamental del realismo. Pero este principio puede sólo ser un principio del realismo si el realismo estuviese ya comprometido con el rechazo a los universales indiscernibles. En efecto, para ver esto con mayor claridad, obsérvese que cualquier realismo que admite universales inexactamente similares mantendrá que compartir universales no es necesario para la similitud de objetos, en la medida que los objetos que tienen universales inexactamente similares pueden por tanto ser similares (aunque no exactamente similares) ${ }^{5}$. Pero entonces, si tener universales inexactamente similares puede conferir similitud inexacta, el fundamento para sostener que los objetos que no comparten universales no pueden ser exactamente similares sólo puede ser el rechazo previo a los universales indiscernibles. Así, no puede fungir como argumento contra los universales indiscernibles el que admitirlos fuese inconsistente con el principio de que los objetos que no comparten universales no son exactamente similares, pues ese principio está basado sólo en el rechazo a los universales indiscernibles.

¿Cuál podría ser la razón para rechazar los universales indiscernibles? No hay nada en el concepto de un universal inmanente que implique que no hay universales indiscernibles. Y la idea de que pueda haber universales indiscernibles inmanentes es perfectamente inteligible. ¿Habrá acaso razones metodológicas? Quizás las teorías que postulan universales indiscernibles son menos simples, menos económicas o menos elegantes que las teorías que no los admiten. Pero el punto de este artículo ha sido sostener que los universales indiscernibles pueden hacer un trabajo útil y por lo tanto proveer ventajas teóricas a las teorías que los postulan. Tal trabajo (por ejemplo, el hecho de que tales teorías son compatibles con la falsedad de la Identidad de Indiscernibles para particulares y que ellas pueden dar cuenta de la similitud entre universales cuantitativos sin caer en algunos de los problemas de la teoría de Armstrong) debe compensar, por lo menos en alguna medida, la presunta falta de simplicidad, economía y elegancia. Si el trabajo hecho por los

\footnotetext{
${ }^{5}$ Armstrong define semejanza de particulares $a$ y $b$ del siguiente modo: "Existe una propiedad $\mathrm{P}$, tal que $a$ tiene $\mathrm{P}$, y existe una propiedad Q, tal que $b$ tiene Q, ya sea que P $=$ Q o P se asemeje a Q" (1978b, 96). Claramente las propiedades en esta definición se supone que son universales y que la semejanza entre ellas es imperfecta o inexacta.
}

Revista de Humanidades de Valparaíso, 2020, No 16, 89-110

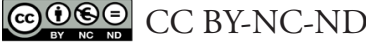


universales indiscernibles puede compensarnos, y por lo tanto deben aceptarse, o si por el contrario la presunta simplicidad, economía o elegancia son preferibles, y por lo tanto deben rechazarse, es algo que debe ser dejado de lado para otra ocasión.

En cualquier caso, podría pensarse que la teoría de los universales indiscernibles es menos simple que la teoría de Armstrong en lo relativo a la definición de semejanza perfecta o exacta entre universales. Armstrong tiene una definición simple de semejanza perfecta o exacta en términos de universales compartidos: $a$ y $b$ se asemejan entre sí exactamente o perfectamente si y sólo si para todos los universales $\mathrm{U}, a$ instancia $\mathrm{U}$ si y sólo si $b$ instancia U (cf. Armstrong, 1978b, 97). ${ }^{6}$ Es decir, dos particulares se asemejan entre sí perfectamente si y sólo si los universales que ellos instancian son numéricamente los mismos. Pero si uno admite universales indiscernibles, entonces la semejanza perfecta entre particulares no es ya solamente un asunto de compartir universales numéricamente idénticos. Una vez que son admitidos los universales indiscernibles, la semejanza perfecta entre particulares se vuelve un asunto disyuntivo: $a$ y $b$ son exactamente o perfectamente semejantes entre ellos si y sólo si los universales que ellos instancian o bien son universales numéricamente idénticos, o bien numéricamente distintos pero universales indiscernibles ("o" inclusivo). De acuerdo a la objeción, la teoría de los universales indiscernibles no provee una posición unificada de la semejanza perfecta de particulares.

Pero la teoría de los universales indiscernibles puede proveer una posición unificada acerca de la semejanza perfecta de particulares. Y, en efecto, esta posición unificada es la base y la motivación para la definición de Armstrong según la cual dos particulares se asemejan entre sí perfectamente si y sólo si los universales que ellos instancian son numéricamente los mismos. Pues la razón por la cual los universales numéricamente idénticos confieren a sus instancias semejanza o similitud perfecta en un respecto es que cada universal se asemeja a él mismo perfectamente por medio de sus instancias (i.e., cada universal es indiscernible de sí mismo). Esto es, el universal U, en cuanto instanciado por el particular $a$, se asemeja perfectamente al universal U, en cuanto instanciado por el particular $b$. Si un universal en cuanto instanciado por un particular pudiera ser cualitativamente diferente de sí mismo en cuanto instanciado por otro particular, aquellos particulares no serían perfectamente semejantes entre ellos en un respecto, a pesar de instanciar numéricamente el mismo universal. La definición de semejanza perfecta de la teoría de los universales indiscernibles es, entonces: $a$ y $b$ se asemejan entre sí exacta o perfectamente si y sólo si para todos los universales $U, a$ instancia $U$ si y sólo si $b$ instancia un universal indiscernible de $\mathrm{U}$ y viceversa.

Otra razón metodológica para rechazar la teoría de los universales indiscernibles podría ser la desarrollada por John Heil, quien piensa que la única ventaja de los universales sobre los tropos es que la primera puede prescindir de la similitud primitiva (cf. 2003,

\footnotetext{
${ }^{6}$ Armstrong pone la definición en términos de propiedades, pero es claro que para él las propiedades son universales.
} 
$159 ; 132)$. Si esto es así, cualquier teoría de universales que no admita universales indiscernibles sería metodológicamente superior a cualquier teoría de universales que postule universales indiscernibles. Pero no es claro que esta sea la única ventaja de los universales sobre los tropos. Por ejemplo, bien podría ser que las teorías de universales provean un punto de vista superior que las teorías de tropos acerca de leyes de la naturaleza (cf. Armstrong 1997, 24). Nótese que una teoría que permite universales indiscernibles, si se sigue el planteamiento de Armstrong de las leyes, podría tener que admitir leyes indiscernibles. De acuerdo con Armstrong, las leyes son universales (cf. Armstrong 1983, 88-90). Supóngase que $F_{1}$ y $F_{2}$ son universales indiscernibles y que $G_{1}$ y $G_{2}$ son otros dos universales indiscernibles, y supóngase que $\mathrm{F}_{1}$ necesita a $\mathrm{G}_{1}$ y que $\mathrm{F}_{2}$ necesita a $\mathrm{G}_{2}$. Entonces $\mathrm{N}\left(\mathrm{F}_{1}, \mathrm{G}_{1}\right)$ y $\mathrm{N}\left(\mathrm{F}_{2}, \mathrm{G}_{2}\right)$ son dos universales, y podría sugerirse que hay dos universales indiscernibles. Podría ser también que las teorías de tropos enfrenten problemas intentando dar cuenta de los truthmakers de ciertas verdades, cosa que no ocurre con las teorías de universales (cf. Armstrong 2005, 310). No estoy diciendo que estoy de acuerdo con Armstrong acerca de esto. Sólo indico que no es obvio que la única ventaja de los universales sobre los tropos sea que las teorías de universales, a diferencia de las de tropos, pueden dispensarse de la similitud primitiva.

Otra objeción para la teoría de universales indiscernibles es que en ella los universales colapsarían en tropos ${ }^{7}$. Pero incluso si todos los universales indiscernibles fuesen instanciados sólo una vez (que no hace falta que sea el caso), aún sería el caso de que ellos podrían haber sido instanciados por muchos particulares diferentes, esto es, ellos podrían haber sido idénticos por medio de sus instancias. Y así es como difieren de los tropos, en la medida que los tropos no podrían haber sido instanciados de manera múltiple, pues éstos pertenecen únicamente al objeto del cual son un tropo (cf. Ehring 2011, 8). En efecto, algunos conciben los tropos como no transferibles, y ello implica que éstos no podrían haber sido instanciados de manera múltiple (cf. Heil 2003, 141). Por supuesto, si los tropos además son ontológicamente dependientes por su identidad de las cosas de las cuales ellos son tropos, esto es, si los tropos no habrían podido ser tenidos por cosas distintas que aquellas que los tienen (cf. Heil 2003, 141-42; Lowe 2006, 97), entonces se agrega otra diferencia entre tropos y universales, ya que un universal podría haber sido instanciado por otros particulares que aquellos que lo instancian ${ }^{8}$.

Puede objetarse que una teoría de universales que postula universales indiscernibles podría socavar un argumento en favor de los universales, y que esto la privaría a sí misma de sustento 9 . Pues un argumento a favor de los universales es que para que los particula-

\footnotetext{
${ }^{7}$ Esta ha sido la objeción más común cuando he sostenido estos planteamientos en conferencias.

${ }^{8}$ Nótese que tanto Heil como Lowe prefieren hablar de modos más que de tropos, pero su razón para preferir aquello no es relevante para el punto que estoy estableciendo aquí. Para mis propósitos, sus modos pueden tratarse como tropos.

${ }^{9}$ Agradezco al evaluador de la versión inglesa de este artículo por notar esta posible objeción.
} 
res sean perfectamente similares en algún respecto debe haber alguna entidad que ellos tengan en común. Pero si hay universales indiscernibles, no es cierto que para que particulares sean perfectamente similares en algún respecto debe haber alguna entidad que ellos tengan en común. Y si eso no es cierto entonces no hay sustento para ninguna teoría de universales.

Sin embargo, esta objeción carece de fuerza, porque el mencionado argumento para la existencia de universales de todos modos tampoco tiene mucha fuerza. Muchas alternativas para la teoría de universales han sido propuestas y todas rechazan la idea de que la similitud perfecta entre particulares precise que estos particulares tengan una entidad en común (y tal rechazo no es una debilidad de tales teorías). En efecto, la existencia de universales tiene mucho mejor sustento si se apela a sus ventajas teóricas sobre sus rivales.

Como se dijo en la Sección 1, nada en el concepto mismo de un universal inmanente implica rechazar los universales indiscernibles. Pero algunos filósofos han definido los universales de tal modo que no podría haber universales indiscernibles. Para ellos, de hecho, lo que distingue los universales de los particulares es precisamente que los últimos pueden ser indiscernibles, mientras que los primeros no. Esto ha sido sugerido por John Wisdom (cf. 1934, 208) y luego por D. C. Williams (cf. 1986, 3). Ni Wisdom, ni Williams proveen ningún argumento para sus tesis; sólo se molestan en aseverarlas. Asimismo, John Heil insinúa esta forma de distinguir particulares y universales cuando dice: "Los objetos particulares pueden ser exactamente parecidos [alike] con respecto a todas sus propiedades (o, al menos, todas sus propiedades intrínsecas) pese a que difieran numéricamente. Los universales son, en contraste, repetibles (cf. Heil 2003, 126). Más recientemente, esa tesis ha sido sostenida por Douglas Ehring (cf. 2011), quien no sólo da un argumento para ella sino que también la elabora y le entrega una formulación minuciosa. Esta es la formulación del criterio de Ehring:

$\mathrm{X}$ es un particular sólo en el caso en que sea posible que exista un $y$ tal que $x$ e $y$ sean no-idénticos pero exactamente similares, y ello independientemente de sus propiedades no intrínsecas, y $x$ es un universal sólo en caso de que no sea posible que exista un $y$ tal que $x$ e $y$ sean no-idénticos pero exactamente similares independiente de sus propiedades no intrínsecas (Ehring 2011, 35).

Ehring argumenta que el criterio hace la distinción correcta (cf. 2011, 32-40), lo compara con otros criterios propuestos para establecer la distinción entre universales y particulares y finalmente concluye que el criterio en términos de indiscernibilidad tiene ventajas sobre los otros (cf. 2011, 40-43). Si esto es así, la teoría de universales indiscernibles es incompatible con el mejor criterio para distinguir entre particulares y universales. Ahora bien, a pesar de que no estoy en desacuerdo con que tal criterio tiene ventajas sobre los otros, creo que tal criterio no puede ser correcto. 
No es claro que tal criterio distinga apropiadamente entre universales y particulares. Por ejemplo, los conceptos son, supuestamente, particulares, y pareciera que ellos satisfacen la identidad de los indiscernibles: ninguna otra cosa que el concepto árbol podría haber sido exactamente similar al concepto árbol. Los números son otro ejemplo: ninguna otra cosa que el número 2 podría haber sido exactamente similar al número 2. (cf. Swinburne 1995, 392).

Se supone, no obstante, que los números y los conceptos son objetos abstractos, y podría pensarse que lo que importa es distinguir entre universales y particulares concretos, y que esto es algo que el criterio hace bien, por lo menos desde un punto de vista extensional si es restringido a particulares concretos. Porque podría pensarse que necesariamente cualquier objeto concreto debe ser posiblemente indiscernible de un otro objeto, mientras que esto no es así con los universales.

Pero de nuevo no es claro que esto sea así, pues podría haber particulares tales que sea necesario que sólo ellos tengan ciertas propiedades. Esto es, podría haber un particular $a$ $\mathrm{y}$ una propiedad $\mathrm{F}$ tales que sea necesario que si cualquier cosa $x$ es $\mathrm{F}, x=a$. En tal caso, el particular $a$ no tendría ningún indiscernible, ya sea en el mismo mundo donde $a$ existe o en cualquier otro. Si Dios es concreto, Dios es plausiblemente uno de esos particulares.

Además, ¿cuál es la razón por la cual debiésemos sólo querer distinguir entre universales y particulares concretos? Es verdad que se supone que los universales representan propiedades escasas (sparse), y las propiedades escasas juegan un rol en dar cuenta de la similitud entre particulares concretos y el poder causal de particulares concretos (cf. Ehring 2011,3). Pero los particulares abstractos también son particulares y entonces lo que hace a un universal un universal no puede ser eso lo que los distingue de los particulares pero no de los particulares abstractos.

Tal vez todo lo que necesitamos es distinguir los universales de aquellos particulares que los instancian. Pero los particulares abstractos también instancian universales (y no existe razón por la que los universales de particulares abstractos sean menos inmanentes que los particulares concretos) ${ }^{10}$. Además, los particulares abstractos pueden tener o no tener relaciones de similitud entre sí. ¿Qué da cuenta de esto? Si los universales pueden dar cuenta de la similitud entre particulares concretos, de seguro ellos pueden también dar cuenta de la similitud entre objetos abstractos. Además, tener los universales que tienen podría ser parte de lo que explica la falta de poderes causales de los objetos abstractos. Algunos universales confieren poderes causales a sus instancias, algunos no. Así, una teoría de universales debe ocuparse no sólo de los universales instanciados por particulares

\footnotetext{
${ }^{10}$ La inmanencia no debe tratarse como si fuese lo mismo que la presencia espaciotemporal en sus instancias. La inmanencia es presencia en sus instancias, y es porque los particulares concretos son localizables espaciotemporalmente que los universales inmanentes también lo son. Pero los particulares abstractos pueden tener universales inmanentes sin éstos estar espaciotemporalmente situados.
} 
concretos, sino también de universales instanciados por particulares abstractos, ya que debería ocuparse de todos los universales. De este modo, lo que una teoría de universales necesita es un criterio para distinguir universales en general de particulares en general.

En pocas palabras, el criterio para distinguir entre particulares y universales en términos de indiscernibilidad no es el correcto. No respetar tal criterio no puede, entonces, sostenerse en contra de una teoría que postule universales indiscernibles. Por supuesto, una teoría de universales que postule universales indiscernibles tendría que proveer un criterio general para distinguir entre particulares y universales. Pero no es parte de este artículo discutir cuál podría ser ese criterio.

\section{Conclusión}

He argüido que postular universales indiscernibles puede resultar útil para una teoría de universales. Nada de lo que he dicho aquí declara o tiene la intención de declarar a la teoría de universales indiscernibles como la mejor teoría de universales. Sin embargo, si lo que he sostenido es correcto, las teorías que postulen universales indiscernibles merecen ulteriores consideración y exploración.

\section{Referencias bibliográficas}

Armstrong, D. M. (1978a). Nominalism and Realism (Vol. 1 of Universals and Scientific Realism). Cambridge: Cambridge University Press.

Armstrong, D. M. (1978b). A Theory of Universals (Vol. 2 of Universals and Scientific Realism). Cambridge: Cambridge University Press.

Armstrong, D. M. (1983). What is a Law of Nature? Cambridge: Cambridge University Press. https://doi.org/10.1017/CBO9781139171700.

Armstrong, D. M. (1997). A World of States of Affairs. Cambridge: Cambridge University Press. https://doi.org/10.1017/CBO9780511583308

Armstrong, D. M. (2005). Four Disputes about Properties. Synthese, 144(3), 309-320. https://doi. org/10.2307/20118566.

Black, M. (1952). The Identity of Indiscernibles. Mind, 61(242), 153-164. https://doi.org/10.1093/ mind/LXI.242.153.

Donnelly, M. (2011). Using Mereological Principles to Support Metaphysics. The Philosophical Quarterly 61(243), 225-246. https://doi.org/10.nn/j.1467-9213.2010.683.x

Eddon, M. (2007). Armstrong on Quantities and Resemblance. Philosophical Studies, 136, 385404. https://doi.org/10.1007/s11098-005-5384-5

Ehring, D. (2011). Tropes. Properties, Objects, and Mental Causation. Oxford: Oxford University Press. https://doi.org/10.1093/acprof:oso/9780199608539.001.0001 
Heil, J. (2003). From an Ontological Point of View. Oxford: Oxford University Press. https://doi. org/10.1093/0199259747.001.0001

van Inwagen, P. (2004). Properties. In T. Crisp, M. Davidson, and D. Vander Laan (eds.) Knowledge and Reality. Essays in Honour of Alvin Plantinga, . Dordrecht: Springer. https:// doi.org/10.1007/1-4020-4733-9

Lewis, D. (1999). 'Against Structural Universals' in his Papers in Metaphysics and Epistemology, 78-107. Cambridge: Cambridge University Press. https://doi.org/10.1017/ CBO9780511625343.004

Loux, M. (1998). Metaphysics. A Contemporary Introduction. London: Routledge. https://doi. org/10.4324/9780203438244

Lowe, J. (2006). The Four-category Ontology. Oxford: Oxford University Press. https://doi. org/10.1093/0199254397.001.0001

Pautz, A. (1997). An Argument against Armstrong's Analysis of the Resemblance of Universals. Australasian Journal of Philosophy, 75(1), 109-111. https://doi. org/10.1080/00048409712347721

Rodriguez-Pereyra, G. (2004). The Bundle Theory is Compatible with Distinct but Indiscernible Universals. Analysis, 64(281), 72-81. https://doi.org/10.1111/j.0003-2638.2004.00463.x

Swinburne, R. (1995). Thisness. Australasian Journal of Philosophy, 73(3), 389-400. https://doi. org/10.1080/00048409512346721

Williams, D. C. (1986). Universals and Existents. Australasian Journal of Philosophy, 64(1), 1-14. https://doi.org/10.1080/00048408612342191

Wisdom, J. (1934). Problems of Mind and Matter. Cambridge: Cambridge University Press. 